\title{
Impact of endoscopic stent insertion on detection of viable circulating tumor cells from obstructive colorectal cancer
}

\author{
SHINYA YAMASHITA ${ }^{1 *}$, MASAHIRO TANEMURA ${ }^{2 *}$, GENTA SAWADA $^{3}$, JEONGHO MOON $^{4}$, YOSUKE SHIMIZU $^{5}$, \\ TOSHIKI YAMAGUCHI ${ }^{5}$, TOSHIO KUWAI ${ }^{5}$, YASUO URATA ${ }^{6}$, KAZUYA KURAOKA ${ }^{5}$, \\ NOBUTAKA HATANAKA $^{7}$, YOSHINORI YAMASHITA ${ }^{5}$ and KIYOMI TANIYAMA ${ }^{5}$ \\ ${ }^{1}$ Department of Surgery, Nissay Hospital, Osaka 565-0871; ${ }^{2}$ Department of Gastroenterological Surgery, \\ Osaka Police Hospital, Osaka 543-0035; ${ }^{3}$ Department of Surgery, Sakai Medical Center, Osaka 593-8304; \\ ${ }^{4}$ Department of Surgery, Osaka Medical Center for Cancer and Cardiovascular Diseases, Osaka 537-8511; \\ ${ }^{5}$ Institute for Clinical Research, National Hospital Organization Kure Medical Center and Chugoku \\ Cancer Center, Hiroshima 737-0023; ${ }^{6}$ Oncolys BioPharma, Inc., Tokyo 106-0032; ${ }^{7}$ Department of \\ Surgery, Japan Community Health Care Organization Osaka Hospital, Osaka 553-0003, Japan
}

Received June 12, 2017; Accepted October 17, 2017

DOI: $10.3892 / \mathrm{ol} .2017 .7339$

\begin{abstract}
The placement of a self-expanding metallic stent (SEMS) in obstructive colorectal cancer (OCRC) is acknowledged to be a safe and effective procedure for the relief of obstruction. However, there is concern that shear forces acting on the tumor during stent expansion may release cancer cells into the circulation, resulting in a poor prognosis. The aim of the present study was to determine whether colonic stent insertion increases viable circulating tumor cells (v-CTCs). A telomerase-specific replication-selective adenovirus-expressing GFP (TelomeScanF35) detection system was used to detect v-CTCs in 8 OCRC patients with a SEMS before and after stent insertion and after surgical resection. In 7 patients, a SEMS was inserted as a bridge to surgery (BTS), and in one patient, a SEMS was inserted for palliation. Surgical resection (R0) was performed in 7 patients. Four patients had no v-CTCs before SEMS placement, two of four measurable patients had an increased number of v-CTCs after SEMS placement (1-3 v-CTCs), and one of two patients with increased v-CTCs developed distant lymphatic metastasis despite curative resection. Four patients
\end{abstract}

Correspondence to: Dr Masahiro Tanemura, Department of Gastroenterological Surgery, Osaka Police Hospital, 10-31 Kitayamacho Tennoujiku, Osaka 543-0035, Japan

E-mail: tanemuram@oph.gr.jp

${ }^{*}$ Contributed equally

Abbreviations: SEMS, self-expanding metallic stent; OCRC, obstructive colorectal cancer; BTS, bridge to surgery; v-CTC, viable circulating tumor cell; TelomeScanF35, OBP-1101, TelomeScanF35

Key words: colorectal cancer, colorectal surgery, endoscopy, stents, intestinal obstruction, circulating tumor cells had v-CTCs (1-19 cells) before SEMS placement, and two of these four patients had an increase in the number of $\mathrm{v}$-CTCs (20-21 cells) after SEMS placement, while one of the four patients died early with distant metastasis. The present study demonstrated that endoscopic stent insertion for OCRC may result in tumor cell dissemination into the peripheral circulation and may induce distant metastases.

\section{Introduction}

At the time of the initial diagnosis of colorectal cancer, $8-13 \%$ of patients have obstructive symptoms $(1,2)$. The treatment of choice for these patients has traditionally been emergency surgery. However, most studies have found that the morbidity and mortality rates are higher for emergency colorectal surgery than for elective surgery, and a temporary colostomy, which decreases patients' quality of life, is needed in many patients, which, in 10-40\% of cases, becomes permanent (3). The purpose of stenting as a bridge to surgery (BTS) is to relieve the acute situation without emergency surgery, allowing elective surgery to be performed and thus improve surgical outcomes, determine the correct tumor stage, detect synchronous lesions, stabilize comorbidities, and carry out laparoscopic surgery. Immediate results in higher surgical risk patients who were stented were better for primary anastomosis, permanent stoma, wound infection, and overall morbidity, resulting in greater benefit. However, stent insertion was recently reported to have a high risk of perforation, re-obstruction, or stent migration $(4,5)$. Moreover, perforations can lead to peritoneal dissemination. Sabbagh et al (6) reported worse overall survival and higher 5-year cancer-specific mortality of left-sided obstructive colorectal cancer (OCRC) patients with self-expanding metallic stent (SEMS) insertion than emergency surgery due to perforation induced by stent insertion. Furthermore, SEMS insertion as a BTS is no longer recommended for symptomatic left-sided OCRC in 
the European Society of Gastrointestinal Endoscopy clinical guidelines due to the high perforation rate (5).

The dissemination of hematogenous tumor cells is an important first step in the metastatic cascade of solid cancers, including colorectal cancer. Given this, detecting and characterizing circulating tumor cells (CTCs) are of major interest to understand how cancer cells migrate through the bloodstream to reach specific distant sites and form metastases. CTC trafficking is poorly understood, and CTCs' biological behavior is not clear. Specific detection of occult metastatic tumor cells at the single-cell stage in the peripheral blood before the occurrence of incurable metastases is now possible due to the development of analytically sensitive immunocytochemical and molecular assays. Several important papers have demonstrated the usefulness of detecting CTCs for the prediction of clinical outcomes in patients with solid tumors, including CRC. The CellSearch ${ }^{\circledR}$ system is the only technology with FDA approval for the detection of CTCs in patients with metastatic colorectal, breast, and prostate cancers (7). This detection system uses positive epithelial cell adhesion molecule (EpCAM) selection of the CTCs. Though most primary CRCs express EpCAM, its expression can be downregulated during tumor cell dissemination due to epithelial-mesenchymal transition (EMT) plasticity (8). Thus, CellSearch may overlook CTCs in cases of tumors that have undergone EMT and lack EpCAM expression. Furthermore, many of the CTCs that are detected by CellSearch are not viable, but apoptotic (9). Currently, no sensitive assays have been developed for the detection of viable CTCs (v-CTCs).

Telomerase expression is a hallmark of cancer (10) and is required for limitless proliferation of tumor cells. The catalytic domain of human telomerase, hTERT, is silenced in normal human somatic cells, but activated in the majority of cancers (11). A new approach for the visual detection of live human CTCs using a telomerase-specific replication-selective adenovirus-expressing GFP has recently been developed (12). The first step was the construction of a GFP-expressing attenuated adenovirus, in which the telomerase promoter regulates viral replication (OBP-401, TelomeScan). However, the identification of false-positive cells (GFP-positive normal blood cells) is a major concern when replicating adenovirus (rAd)-GFP is used, particularly at high titers. Furthermore, rAd-GFP cannot detect CTCs lacking or expressing low levels of coxsackievirus-adenovirus receptor (CAR), because rAd-GFP is constructed based on Ad serotype 5, which recognizes CAR. In order to avoid the identification of false-positive cells, sequences that are perfectly complementary to blood cell-specific microRNA, miR-142-3p, were incorporated into the 3'-untranslated region of the E1B and GFP genes. In addition, the fiber protein was replaced with that of Ad serotype 35, which recognizes human CD46, creating rAdF35-142T-GFP (OBP-1101, TelomeScanF35). With this TelomeScanF35, not only CAR-positive cancer cells, but also CAR-negative cancer cells were efficiently labeled with GFP (13).

Based on the literature examining the strong and weak points of stent insertion for OCRC patients, suspicion has been raised about whether shear forces that act on the tumor during expansion of the stent may result in the release of CTCs into the circulation and induce early distant metastases, resulting in a poor prognosis despite curative resection for OCRC. The present study aimed to clarify whether insertion of colonic stents increased v-CTC levels using a novel detection method, TelomeScanF35, and to address whether colonic stenting for OCRC is friend or foe.

\section{Materials and methods}

Patients. Between October 2013 and June 2015, patients who underwent stenting for OCRC were enrolled. Stenting was performed in patients needing emergency bowel decompression for OCRC, excluding those with suspected or impending perforation, enteral ischemia, or intra-abdominal abscess. This study was a single institution trial, approved by the Kure Medical Center IRB (25-40) prior to enrolling patients and was conducted in accordance with the Declaration of Helsinki and Good Clinical Practice. Written informed consent was obtained from all patients prior to any study procedure or treatment. Clinicopathological factors and clinical stage were classified using the criteria of the Union for International Cancer Control (UICC) (14). Patients with primary tumors who were undergoing resection were classified as 'BTS', while patients who were not scheduled for surgery were classified as 'palliative'. All clinical data were collected prospectively, and all patients who underwent stent placement were followed-up after discharge.

Inclusion criteria. The inclusion criterion was large bowel obstruction diagnosed by abdominal X-ray, colonoscopy, or computed tomography (CT) scan, including those with colorectal obstruction secondary to malignant neoplasms. Only patients with no previous colonic stenting were included.

Colonic stenting. SEMS placement was performed by one of two experienced endoscopists (T.K., T.Y.) at Kure Medical

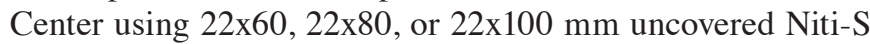
stents (Taewoong Medical Co., Ltd., Seoul, South Korea) in all cases. After the obstruction was confirmed by CT imaging, an endoscope was passed to the obstruction. The stricture was passed with a tandem catheter and JAG wire under fluoroscopic guidance; contrast was injected to delineate the stricture and confirm intraluminal deployment. After stent deployment, its position was confirmed by fluoroscopic imaging. Successful deployment with fluoroscopic confirmation was defined as technical success, while clinical success was defined as the resolution of obstructive symptoms with stool/flatus passage. Abdominal X-rays were taken one day after stenting to further confirm stent extension and position. Finally, clinical success was defined as the relief of obstructive symptoms, while technical success was defined as satisfactory dilatation of the stenosis.

Surgical procedures. Patients undergoing resection after SEMS placement as a BTS were the primary subjects. BTS was defined as scheduled elective surgery regardless of the time elapsed between SEMS insertion and surgery. In a BTS setting, laparoscopic surgery was performed.

Assessment of tumor stage and oncological management. Tumor stage was defined using the TNM classification of Malignant Tumors 7th edition, published by the UICC. The 


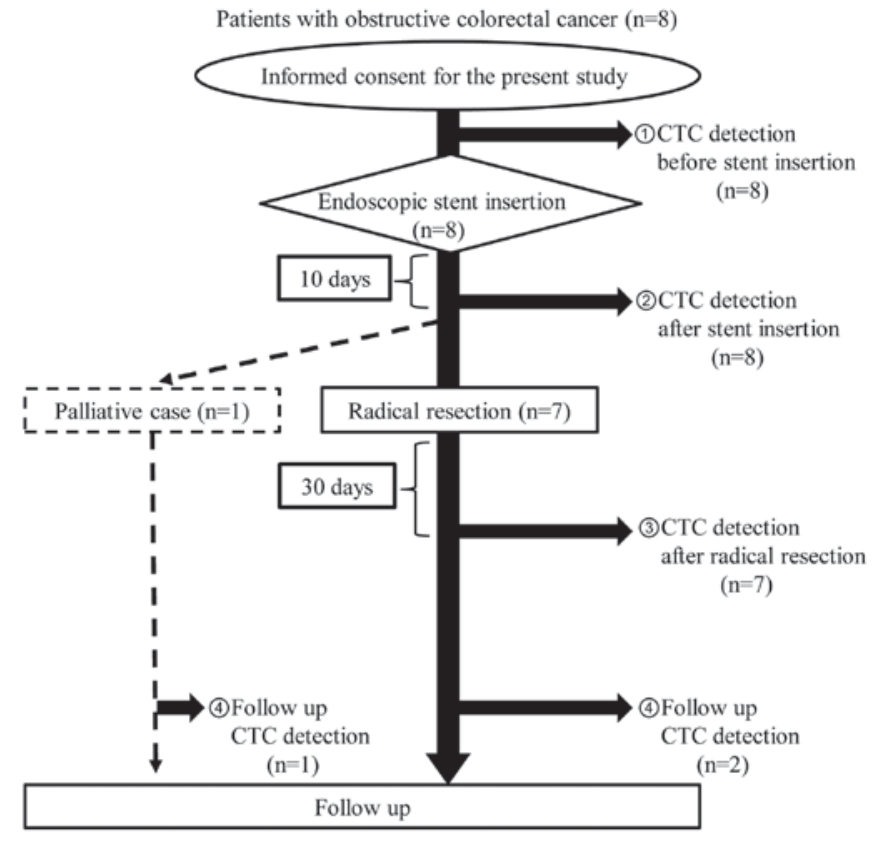

Figure 1. Flowchart of this study. Eight patients with OCRC were enrolled. SEMS insertion was performed for 7 patients as a BTS and for 1 patient as palliative therapy. CTC detection by TelomeScanF35 was conducted before/after stent insertion and after surgical resection. Three of eight patients also underwent CTC detection in the follow-up period. CTC, circulating tumor cells; OCRC, obstructive colorectal cancer; SEMS, self-expanding metallic stent.

final oncological strategy was decided based on discussion in a weekly, multidisciplinary staff meeting. Patients with UICC stage II and III cancers were advised to undergo adjuvant chemotherapy. The chemotherapy protocol in stage II CRC with risk factors for recurrence (46-1201) was set by the Japanese Foundation for Multidisciplinary Treatment of Cancer (JFMC, http://www.jfmc.or.jp/).

Detection of CTCS in the blood samples of OCRC. Peripheral blood was collected for CTC evaluation before/after SEMS placement and after resection. CTCs in the peripheral blood samples of cancer patients were detected as previously described $(12,13)$. Briefly, cells recovered from the 7.5-ml blood samples of OCRC patients were incubated with $1 \times 10^{9} \mathrm{VP}$ of rAdF35-142T-GFP (TelomeScanF35) at $37^{\circ} \mathrm{C}$. Following 24-h incubation, the cells were washed and stained with anti-human CD45 antibody (clone HI30; Biolegend, San Diego, CA) and then observed by fluorescence microscopy. Human v-CTCs and false-positive cells were defined as $\mathrm{GFP}^{+} / \mathrm{CD}^{2} 5^{-}$and $\mathrm{GFP}^{+} / \mathrm{CD}^{2} 5^{+}$cells, respectively. To distinguish v-CTCs as of epithelial origin, cells were stained with anti-cytokeratin antibody (628502 and 628602; BioLegend, San Diego, CA, USA). To further distinguish v-CTCs as of mesenchymal origin, cells were labeled with anti-vimentin antibody (Ab45939; Abcam, Cambridge, UK).

\section{Results}

Patients' characteristics. From October 2013 to June 2015, 8 patients were enrolled. A flowchart of these 8 patients is shown in Fig. 1. There were no exclusions as a result of loose stenosis on colonoscopy, adhesive small bowel obstruction, or placement of another type of SEMS. Stent insertion was performed as a BTS in 7 patients and for palliation in one patient. CTC detection by TelomeScanF35 was performed in all 8 patients at the times described above.

Table I lists the patients' baseline characteristics. The median age was 76 years (range, 62-85 years). Five patients had an Eastern Cooperative Oncology Group performance status of 0 , and the remaining 3 patients had performance status of 1 or 2 (PS1 1, PS2 2). OCRCs were located in the sigmoid colon in 5 patients, descending colon in 2 patients, and transverse colon in 1 patient. The median tumor size was $70 \mathrm{~mm}$ (range, $40-80 \mathrm{~mm}$ ). Four patients were diagnosed as TNM stage II, and 4 patients were diagnosed as stage III. The median pre-operative CEA level was $8.3 \mathrm{ng} / \mathrm{ml}$ (range, $3.4-91.4 \mathrm{ng} / \mathrm{ml})$.

Technical success of SEMS placements. All patients underwent successful SEMS insertion. All patients required a single stent in the first attempt. Of the total of 8 stents, the most commonly used stent lengths were $22 \times 80$ and $22 \times 100 \mathrm{~mm}(\mathrm{n}=3)$, but in 2 patients, a $22 \times 60-\mathrm{mm}$-long stent was selected.

Analysis of clinical success as a BTS and perioperative complications. All 7 patients in the BTS group underwent laparoscopic surgery. No patients required a permanent stoma. The median time from SEMS placement to surgery was 15 days (range 13-27 days). No silent perforations occurred. As to perioperative complications, there was no anastomotic leakage. Additionally, no surgical site infections (SSIs) of any grade occurred. Taken together, these findings indicate that there was no correlation between the time elapsed from stent insertion to surgery and perioperative complications, including anastomotic leakage and SSI. The median duration of hospitalization after surgery was 10 days (range, 7-15 days). Both the overall 30-day mortality rate after technically successful SEMS placement and hospital postoperative mortality were zero.

Survival. Median follow-up in the 8 patients with SEMS placement was 28.1 months (range, 15.5-34.6 months). There was one cancer-related death in the palliative patient, and disease recurrence occurred in one patient in the BTS group 24 months after curative resection (Table II). The site of recurrence in this patient was pelvic lymph nodes (Fig. 2), but this recurrent patient had undergone $\mathrm{R} 0$ resection and received systemic chemotherapy with UFT+LV after resection (Table II).

Detection of CTCs in the blood isolated from OCRC patients with SEMS placement. The TelomeScanF35 system assumes that GFP-positive and CD45-negative cells are true CTCs. During cancer invasion and metastasis, some cancer cells undergo an EMT (15), resulting in the downregulation of some cytokeratins (16), and, therefore, these important CTCs will be stained with both anti-vimentin antibody and anti-cytokeratin antibody to prevent missing these CTCs. As shown in Table II, the number of CTCs detected within the peripheral blood circulation before/after SEMS placement and after resection was summarized. Prior to SEMS placement, CTCs were detected in the peripheral blood (range, 1-19 CTCs) in 50\% 
Table I. Clinicopathological factors, surgical methods and complications in 8 patients undergoing SEMS insertion.

\begin{tabular}{lc}
$\begin{array}{l}\text { Clinicopathological characteristics, } \\
\text { surgical methods and complications }\end{array}$ & No. \\
\hline Number of patients & 8 \\
Sex (male/female) & $5 / 3$ \\
Age (median, range) & $76,62-85$ \\
Performance status (0/1-2) & $5 / 3$ \\
Pre-operative CEA level (ng/ml, median, range) & $8.3,3.4-91.4$ \\
Tumor location (transverse/descending/sigmoid) & $1 / 2 / 5$ \\
Tumor stage (T2/T3/T4) & $0 / 8 / 0$ \\
Node status (N0/N1-2) & $4 / 4$ \\
UICC stage (II/III) & $4 / 4$ \\
Maximal tumor size (mm, median, range) & $70,40-80$ \\
Surgical method (Open surgery/laparoscopic & $0 / 7$ \\
surgery) & \\
Curability (R0/R1-2) & $7 / 0$ \\
Colostomy formation (yes/no) & $0 / 7$ \\
Post operative complication (yes/no) & $0 / 7$ \\
Histopathological type (Well/Mod/Por/Muc) & $4 / 3 / 0 / 1$ \\
Venous invasion (+/-) & $2 / 5$ \\
Lymphatic invasion (+/-) & $2 / 5$
\end{tabular}

${ }^{a}$ Well, well-differentiated adenocarcinoma; Mod, moderatelydifferentiated adenocarcinoma; Por, poorly-differentiated adenocarcinoma; Muc, mucinous adenocarcinoma; CEA, carcinoembryonic antigen; UICC, Union for International Cancer Control; SEMS, self-expanding metallic stent.

$(4 / 8)$ of the patients. Following stent insertion, a large increase in the number of CTCs was seen in two of these four patients. On the other hand, no CTCs were detected in four of eight patients before stent insertion, but, following stent insertion, an increase in the number of CTCs was seen in two of four patients in whom no CTCs were detected before stent insertion. However, no significant differences in the number of CTCs before/after stent insertion were observed, because of the small study size. The majority of v-CTCs detected by TelomeScanF35 expressed the vimentin molecule, which is a mesenchymal marker (EMT marker) on the cell surface. It is important to note that v-CTCs, expressing both vimentin and cytokeratin molecules (double-positive CTCs) appeared in the peripheral blood circulation after SEMS insertion in case 1 (Table II). Unfortunately, early recurrence occurred in case 1 despite curative resection and adjuvant chemotherapy (Table II, Fig. 2). Taken together, it was clearly demonstrated that an increase in the number of CTCs was observed after stent insertion, and that shear forces that act on the tumor during expansion of the SEMS may result in a direct release of some cancer cells, undergoing or not undergoing EMT in the peripheral blood circulation, and this may induce early recurrence despite $\mathrm{R} 0$ resection or adjuvant therapy. One elderly patient was treated as a palliative case, as described above. As shown in Fig. 3, 3 cells of vimentin-positive v-CTCs were detected before stent insertion, the morphological findings of these detected v-CTCs were single-cell, and they were similar to the images of peripheral blood cells. Subsequent to stent insertion, the number of v-CTCs was markedly increased, and the phase-contrast and fluorescent images of v-CTCs displayed the cell-cluster formation. Accordingly, the large cancer cell-clusters were squeezed into the peripheral blood circulation by the mechanical destruction of tumor vascular networks during SEMS expansion. Furthermore, these cell-clusters of $\mathrm{v}$-CTCs were continuously detected for over one year after stent insertion (Fig. 3).

\section{Discussion}

Acute malignant colorectal obstruction occurs in $8-13 \%$ of CRC patients $(1,2)$. BTS SEMS placement has been shown to of use in the surgical treatment of patients with OCRC (17). However, not all patients achieve the same benefit, because older patients or those with important comorbidities are the ones who would benefit most from transforming emergency surgery into elective surgery. Moreover, obstructive colitis caused by OCRC sometimes involves skipped lesions (18), and identifying mucosal changes in the whole colon is difficult by verifying the resected stump and specimens. These mucosal changes are considered to increase the risk of anastomotic leakage associated with obstructive colitis. Accordingly, OCRC patients with stenting as a BTS should be able to undergo preoperative colonoscopy to evaluate mucosal changes, including obstructive colitis and mucosal edema. Furthermore, recent noteworthy papers reported that stent insertion is now safe due to better techniques, improved training and experience of the physicians who perform this procedure, and clearer guidelines $(17,19,20)$.

However, whether stenting as a BTS is oncologically safe in the management of acute malignant colonic obstruction must be considered. Theoretically, enforced radical dilatation by a stent could not only increase the risk of perforation, but tumor manipulation can result in cancer cell spread into the surrounding lymphatic vessels and peripheral bloodstream $(4,21,22)$. Accordingly, in the present study, it was found that a markedly increased number of v-CTCs detected by a novel method, TelomeScanF35 (13), was found in patients' circulation after completion of endoscopic stent insertion. Moreover, serial measurement of v-CTCs before/after stent insertion and after radical resection was performed to evaluate in which patients the v-CTCs are effectively cleared from the blood by either radical resection or adjuvant chemotherapy.

As shown in Table II, v-CTCs were detected in the peripheral circulation in four (BTS: 3 patients, palliative: 1 patient) of eight patients prior to stent placement, and either a potent increase or a similar level of the marker of v-CTCs was observed in two of these four patients after stent insertion, except for the one palliative patient. However, curative resection of primary tumor and adjuvant chemotherapy may lead to significant clearance of v-CTCs, which may be associated with no recurrence. On the other hand, no v-CTCs were detected in four of eight patients before stent insertion, and subsequently, $\mathrm{v}$-CTCs were detected in two of these four patients after stent placement. For these two patients who were v-CTC-positive, one patient unfortunately had early relapse despite curative resection, adjuvant treatment, and a normalized CEA level. 


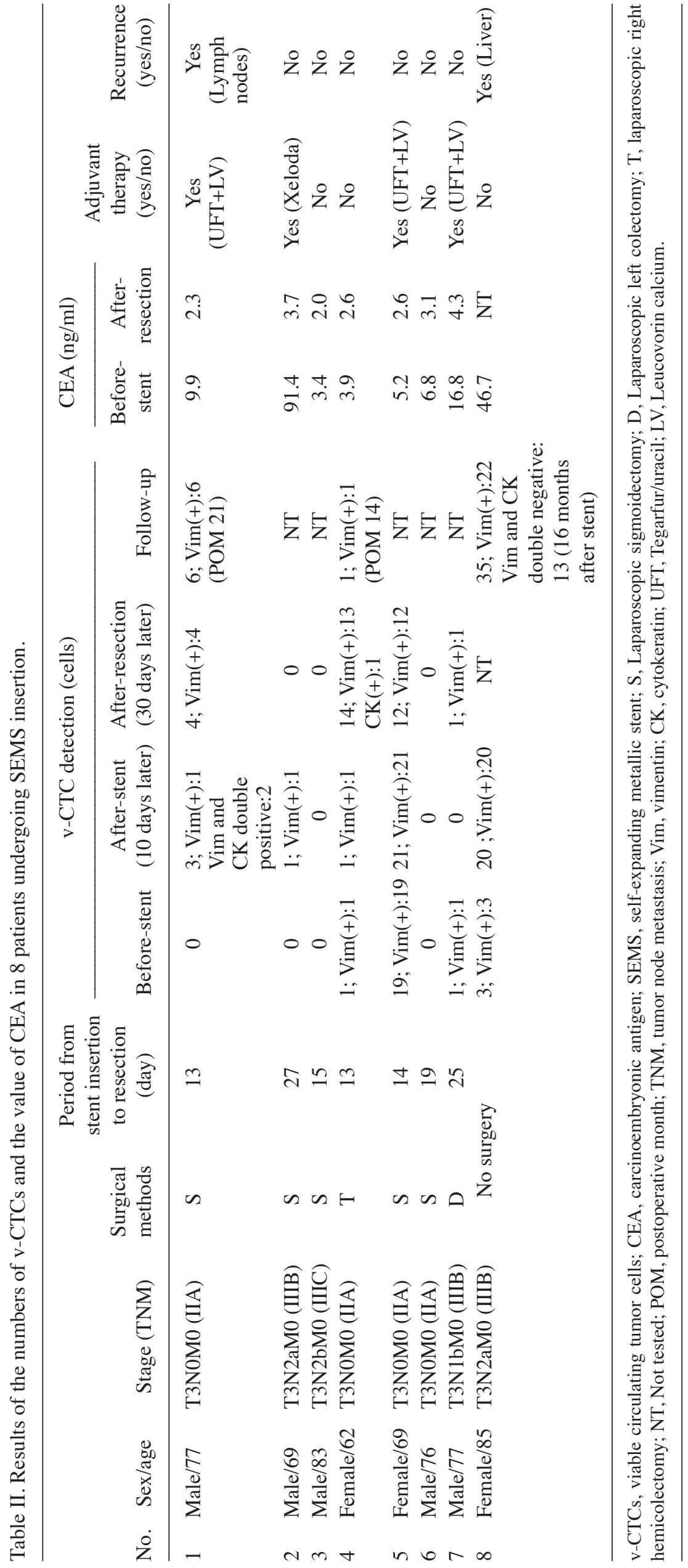


A
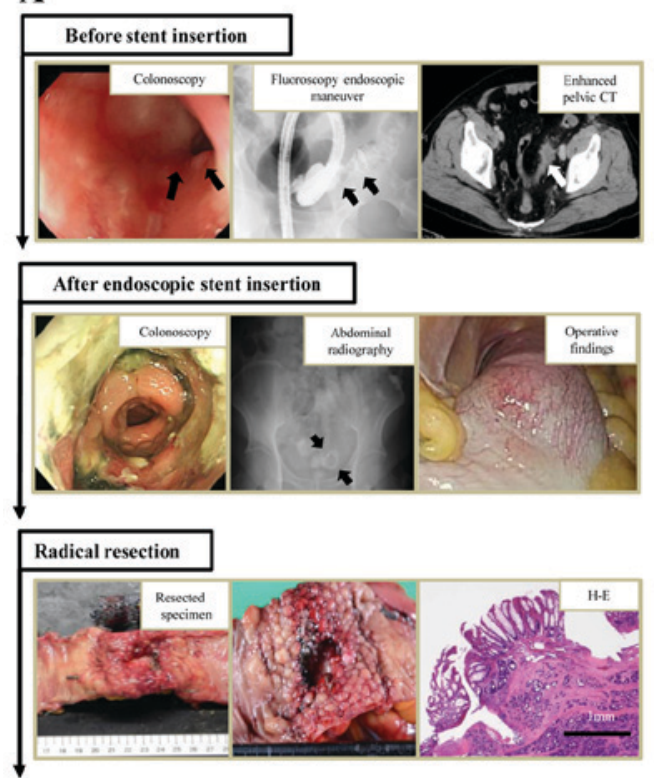

Follow-up CT and PET-CT

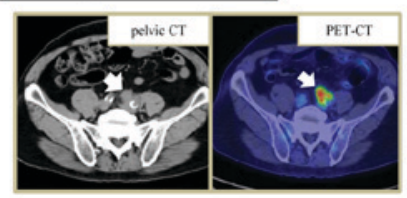

B

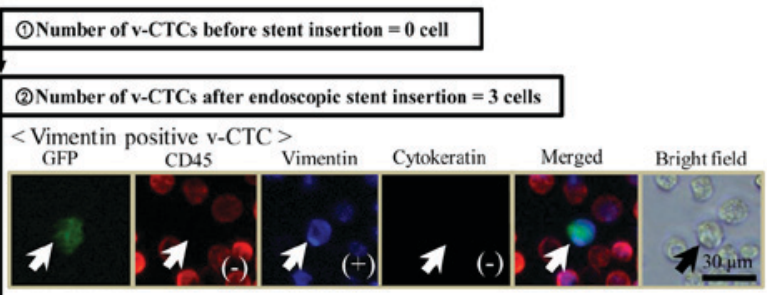

$<$ Vimentin and cytokeratin double positive $\mathrm{v}$-CTC $>$

GFP CD45 Vimentin Cytokeratin Merged Bright field
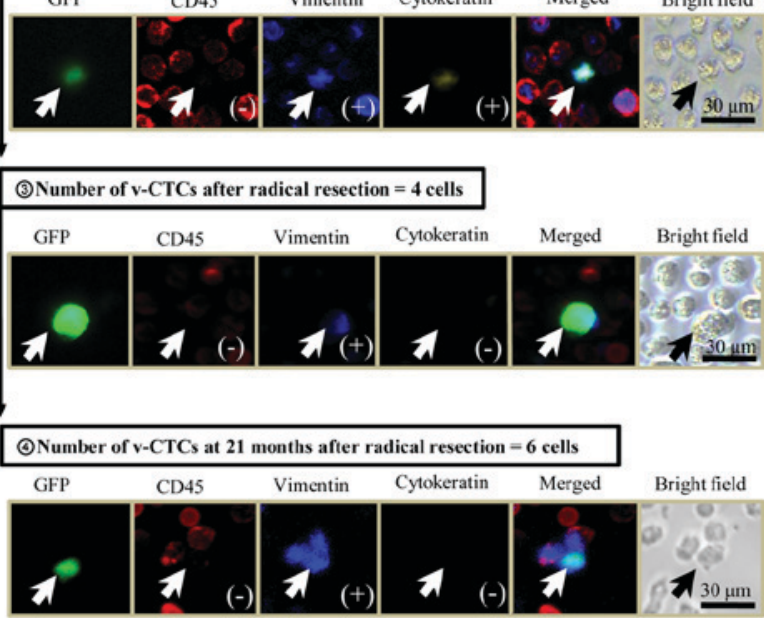

Figure 2. The clinical course and number of v-CTCs of the patient with recurrence in pelvic lymph nodes after surgery (case 1). (A) Black and white arrows show stenosis due to colorectal cancer before stent insertion, a SEMS after endoscopic stent insertion and recurrence in the pelvic lymph nodes at follow-up CT and PET-CT. (B) White and black arrows show v-CTCs at each point of the clinical course. v-CTCs continue to be observed after radical resection. GFP, green fluorescent protein; CD, cluster of differentiation; v-CTC, viable circulating tumor cells; PET, positron emission tomography; CT, computed tomography; $\mathrm{H} \& \mathrm{E}$, hematoxylin and eosin.
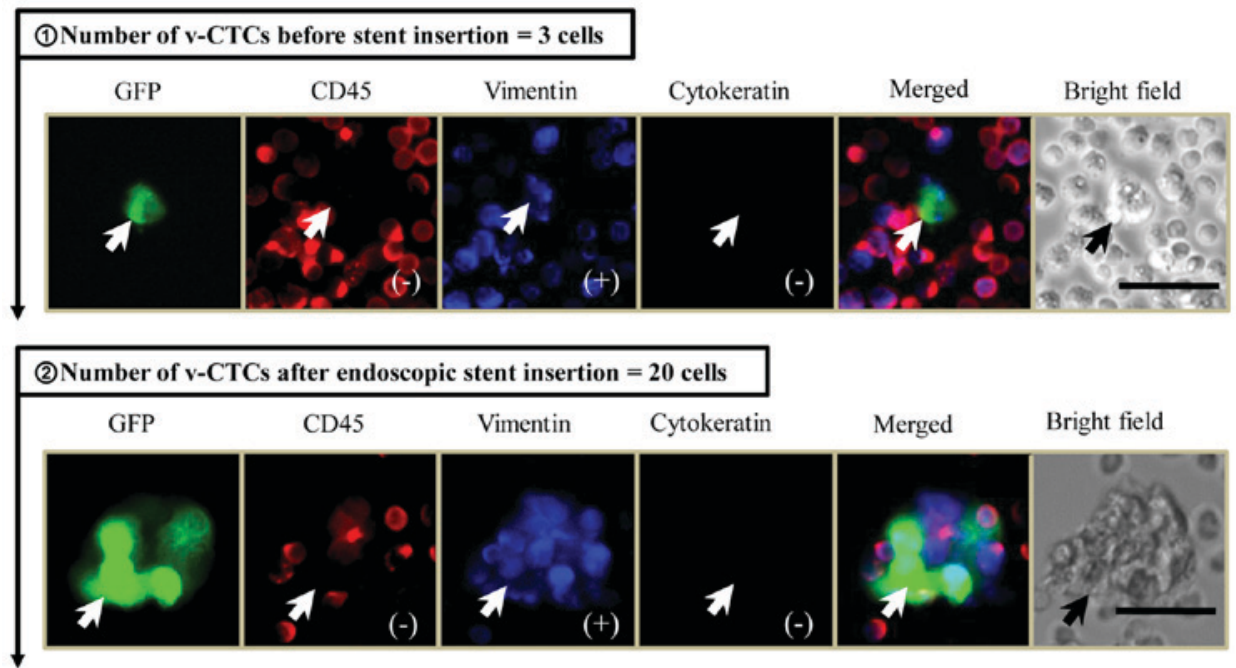

(4) Number of $\mathrm{v}$-CTCs at 16 months after stent insertion $=\mathbf{3 5}$ cells

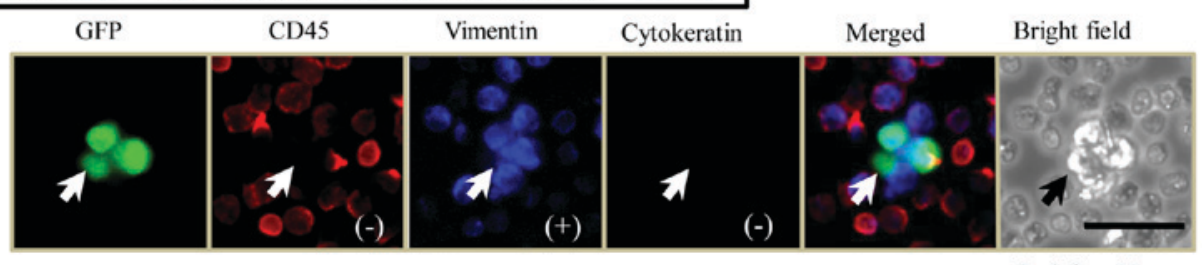

Figure 3. The number of v-CTCs in the patient given palliative therapy (case 8). v-CTCs are single-cell before stenting, and a cell-cluster of v-CTCs forms after stenting. They continue to appear for over one year after stenting. GFP, green fluorescent protein; CD, cluster of differentiation; v-CTC, viable circulating tumor cells. 
The number of v-CTCs was further increased after resection and in the follow-up period. In particular, the detected v-CTCs expressed vimentin, which is a mesenchymal marker. Many v-CTCs probably stay detected as vimentin-positive cells due to an EMT process of tumor cells (15). This group of CTCs may be important in the treatment of metastatic disease, because they are stem-like cancer cells that appear to not respond well to current therapies (23). The other patient developed no recurrence despite the advanced stage of the primary CRC. These clinical findings may be associated with elimination of $\mathrm{v}$-CTCs after surgery. Normally, the malignant potential of these v-CTCs is not very strong, and, consequently, these cells cannot survive long in the blood circulation.

In the present series of patients with stent placement, it was not possible to investigate whether there was a significant correlation between CTCs and tumor stage, histopathological type of tumor, venous invasion, or lymphatic invasion. Thus, stenting's prognostic impact continues to be unclear. Even though stenting is beneficial for OCRC, there are still oncological issues that need to be clarified. Recently, one retrospective study showed that the incidence of perineural invasion was significantly increased after stenting (22). Perineural invasion is known to be a marker of a poor prognosis in colorectal cancer (24). The reason why stenting appears to induce perineural invasion is not known, though it may be related to the pressure effect of a self-expandable stent, which induces cancer cell invasion into the perineural space. In a similar mechanism, cancer cells could be pushed into the surrounding vessels and into the peripheral bloodstream, resulting in CTC detection after stenting.

In conclusion, this is the first report that supports the hypothesis of a correlation between an increase in v-CTCs, serially detected by TelomeScanF35, and stent placement in OCRC patients. The conclusions of this study may open a window of opportunity for raising an alarm about SEMS placement in OCRC. Although stenting has some advantages as a BTS in OCRC, the oncological risk and long-term prognosis of this approach have not been clarified. In the future, a large randomized, controlled study of stenting as a BTS is needed to clarify its oncological safety, feasibility, and long-term prognosis.

\section{Acknowledgements}

The authors would like to thank Mrs. Mizuki Nishimura and Mrs. Kiyomi Yagyu from the Institute for Clinical Research, National Hospital Organization Kure Medical Center and Chugoku Cancer Center (Hiroshima, Japan) for outstanding technical assistance.

This study was supported by a grant from the Ministry of Education, Sports and Culture of Japan to M. T. (no. 16K10618).

\section{References}

1. Winner M, Mooney SJ, Hershman DL, Feingold DL, Allendorf JD, Wright JD and Neugut AI: Incidence and predictors of bowel obstruction in elderly patients with stage IV colon cancer: A population-based cohort study. JAMA Surg 148: 715-722, 2013.

2. Jullumstrø E, Wibe A, Lydersen S and Edna TH: Colon cancer incidence, presentation, treatment and outcomes over 25 years. Colorectal Dis 13: 512-518, 2011

3. Deans GT, Krukowski ZH and Irwin ST: Malignant obstruction of the left colon. Br J Surg 81: 1270-1276, 1994.
4. Datye A and Hersh J: Colonic perforation after stent placement for malignant colorectal obstruction-causes and contributing factors. Minim Invasive Ther Allied Technol 20: 133-140, 2011.

5. van Hooft JE, van Halsema EE, Vanbiervliet G, Beets-Tan RG, DeWitt JM, Donnellan F, Dumonceau JM, Glynne-Jones RG, Hassan C, Jiménez-Perez J, et al: Self-expandable metal stents for obstructing colonic and extracolonic cancer: European society of gastrointestinal endoscopy (ESGE) clinical guideline. Endoscopy 46: 990-1053, 2014.

6. Sabbagh C, Browet F, Diouf M, Cosse C, Brehant O, Bartoli E, Mauvais F, Chauffert B, Dupas JL, Nguyen-Khac E and Regimbeau JM: Is stenting as 'a bridge to surgery' an oncologically safe strategy for the management of acute, left-sided, malignant, colonic obstruction? A comparative study with a propensity score analysis. Ann Surg 258: 107-115, 2013.

7. Cristofanilli M, Budd GT, Ellis MJ, Stopeck A, Matera J, Miller MC, Reuben JM, Doyle GV, Allard WJ, Terstappen LW and Hayes DF: Circulating tumor cells, disease progression, and survival in metastatic breast cancer. N Engl J Med 351: 781-791, 2004.

8. Tsai JH and Yang J: Epithelial-mesenchymal plasticity in carcinoma metastasis. Genes Dev 27: 2192-2206, 2013.

9. Denève E, Riethdorf S, Ramos J, Nocca D, Coffy A, Daurès JP, Maudelonde T, Fabre JM, Pantel K and Alix-Panabières C: Capture of viable circulating tumor cells in the liver of colorectal cancer patients. Clin Chem 59: 1384-1392, 2013.

10. Hanahan D and Weinberg RA: Hallmarks of cancer: The next generation. Cell 144: 646-674, 2011.

11. Cong YS, Wright WE and Shay JW: Human telomerase and its regulation. Microbiol Mol Biol Rev 66: 407-425, 2002.

12. Kojima T, Hashimoto Y, Watanabe Y, Kagawa S, Uno F, Kuroda S, Tazawa H, Kyo S, Mizuguchi H, Urata Y, et al: A simple biological imaging system for detecting viable human circulating tumor cells. J Clin Invest 119: 3172-3181, 2009.

13. Sakurai F, Narii N, Tomita K, Togo S, Takahashi K, Machitani M, Tachibana M, Ouchi M, Katagiri N, Urata Y, et al: Efficient detection of human circulating tumor cells without significant production of false-positive cells by a novel conditionally replicating adenovirus. Mol Ther Methods Clin Dev 3: 16001, 2016.

14. Sobin LH and Fleming ID: TNM Classification of Malignant Tumors, fifth edition (1997). Union Internationale Contre le Cancer and the American Joint Committee on Cancer. Cancer 80: 1803-1804, 1997.

15. Heerboth S, Housman G, Leary M, Longacre M, Byler S, Lapinska K, Willbanks A and Sarkar S: EMT and tumor metastasis. Clin Transl Med 4: 6, 2015.

16. Lamouille S, Xu J and Derynck R: Molecular mechanisms of epithelial-mesenchymal transition. Nat Rev Mol Cell Biol 15: 178-196, 2014.

17. Park SJ, Lee KY, Kwon SH and Lee SH: Stenting as a bridge to surgery for obstructive colon cancer: Does it have surgical merit or oncologic demerit? Ann Surg Oncol 23: 842-848, 2016.

18. Chang HK, Min BS, Ko YT, Kim NK, Kim H and Cho CH: Obstructive colitis proximal to obstructive colorectal carcinoma. Asian J Surg 32: 26-32, 2009.

19. Saito S, Yoshida S, Isayama H, Matsuzawa T, Kuwai T, Maetani I, Shimada M, Yamada T, Tomita M, Koizumi K, et al: A prospective multicenter study on self-expandable metallic stents as a bridge to surgery for malignant colorectal obstruction in Japan: Efficacy and safety in 312 patients. Surg Endosc 30: 3976-3986, 2016.

20. Shimizu Y, Tominaga H, Yamashita S, Kimura Y, Odagiri K, Kurokawa T, Yamaguchi M, Takahashi G, Sawada G, Inoue M, et al: Usefulness of metallic stent for left-sided obstructive colon cancer. Gan To Kagaku Ryoho 42: 2236-2238, 2015 (In Japanese).

21. Maruthachalam K, Lash GE, Shenton BK and Horgan AF: Tumour cell dissemination following endoscopic stent insertion. Br J Surg 94: 1151-1154, 2007.

22. Haraguchi N, Ikeda M, Miyake M, Yamada T, Sakakibara Y, Mita E, Doki Y, Mori M and Sekimoto M: Colonic stenting as a bridge to surgery for obstructive colorectal cancer: Advantages and disadvantages. Surg Today 46: 1310-1317, 2016.

23. Yang MH, Imrali A and Heeschen C: Circulating cancer stem cells: The importance to select. Chin J Cancer Res 27: 437-449, 2015.

24. Knijn N, Mogk SC, Teerenstra S, Simmer F and Nagtegaal ID: Perineural invasion is a strong prognostic factor in colorectal cancer: A systematic review. Am J Surg Pathol 40: 103-112, 2016. 